\title{
BMJ Open Effects of smoking, gender and occupational exposure on the risk of severe pulmonary fibrosis: a population-based case-control study
}

\author{
Magnus Ekström, ${ }^{1,2}$ Torbjörn Gustafson, ${ }^{3}$ Kurt Boman, ${ }^{3}$ Kenneth Nilsson, ${ }^{3}$ \\ Göran Tornling, ${ }^{4}$ Nicola Murgia, ${ }^{5}$ Kjell Torén ${ }^{6}$
}

To cite: Ekström M, Gustafson T, Boman $\mathrm{K}$, et al. Effects of smoking, gender and occupational exposure on the risk of severe pulmonary fibrosis: a population-based casecontrol study. BMJ Open 2014;4:e004018. doi:10.1136/bmjopen-2013004018

- Prepublication history for this paper is available online. To view these files please visit the journal online (http://dx.doi.org/10.1136/ bmjopen-2013-004018).

Received 13 September 2013 Revised 18 November 2013 Accepted 28 November 2013

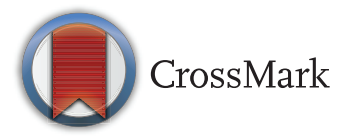

For numbered affiliations see end of article.

Correspondence to Dr Magnus Ekström; pmekstrom@gmail.com

\section{ABSTRACT}

Objectives: To estimate the effects of smoking, gender and occupational exposure on the risk of developing severe pulmonary fibrosis (PF), including dose-response and interaction effects.

Methods: National case-control study of 171 patients (cases) who had started a long-term oxygen therapy for PF in Sweden between February 1997 and April 2000, and 719 random control participants from the general population. Of these cases, 137 had probable idiopathic PF (IPF). The ORs for smoking, gender and occupational exposure were estimated using MantelHaenszel analysis and conditional logistic regression, controlling for age and year of diagnosis.

Results: The adverse effect of smoking was amplified by male gender and occupational exposure, OR 4.6 (95\% Cl 2.1 to 10.3) for PF, and OR 3.0 (1.3 to 6.5) for IPF, compared with in non-exposed women. Higher cumulative smoking exposure was linearly associated with increased risks. Compared with smoking less than 10 pack-years, smoking $\geq 20$ pack-years was associated with increased risk of PF and IPF, OR 2.6 (1.4 to 4.9 ) and OR 2.5 (1.3 to 5.0), respectively.

Conclusions: Smoking has a dose-related association with increased risk of severe PF. Men with a history of smoking and occupational exposure is a particular risk group for developing severe PF.

\section{BACKGROUND}

Pulmonary fibrosis (PF) constitutes a range of conditions that are either secondary to diseases or factors, such as systemic inflammatory disease and drug therapies, or primary, of which the most common is idiopathic $\mathrm{PF}$ (IPF). ${ }^{1}$

IPF is a chronic, progressive, fibrosing interstitial lung disease with a high risk of rapid progression and mortality. ${ }^{2}$ Median survival after diagnosis is approximately 2 years. ${ }^{2}$ The incidence of IPF has increased over time, with marked regional differences suggesting the pathogenic role of various environmental and occupational exposures. ${ }^{3}$

\section{Strengths and limitations of this study}

Population-based case-control study of participants developing oxygen-dependent pulmonary fibrosis in Sweden with randomly selected controls from the general population.

- Analysis of detailed exposure data, accounting for confounders and lag time between exposure and disease.

- As the underlying aetiology may be difficult to ascertain in patients with oxygen-dependent pulmonary fibrosis, idiopathic pulmonary fibrosis was defined through review of national administrative register data and individual medical records.

The aetiology of IPF remains unknown. ${ }^{2}$ The majority of patients with IPF are men with a history of current or past smoking. ${ }^{2}$ Most case-control studies have suggested an association between smoking and an increased risk of $\mathrm{IPF}^{4-7}$ although one study reported no such association. ${ }^{8}$ This inconsistency is most likely due to differences in study settings, the included covariates and, in some studies, the use of hospital patients as control participants, which might have biased the smoking estimates. ${ }^{4}$ 9-11 Occupational exposures, including metal, stone and wood dust, have been linked to higher risks of developing IPF. ${ }^{12}$

It is unknown whether there are interactions between smoking, gender, and occupational exposure and the risk of developing IPF. The only two studies which analysed interactions reported a tendency towards an amplified IPF risk in patients with a history of smoking and occupational exposure, but the studies failed to establish statistically significant interactions. ${ }^{6}{ }^{13}$ Moreover, it is unclear whether smoking really is an aetiological factor for IPF, as studies included low 
numbers of patients, and data on whether there is a dose-dependent relation between smoking and the risk of IPF are limited.

The aim of the present nationwide case-control study was, therefore, to estimate the associations between smoking, gender, occupational exposure and the risk of developing severe $\mathrm{PF}$.

\section{METHODS}

This was a national, register-based case-control study. The patients who had started a long-term oxygen therapy (LTOT) for PF between 1 February 1997 and 4 April 2000 in the national Swedevox register were eligible for inclusion as cases. The Swedevox register covers $85 \%$ of all patients starting LTOT in Sweden. ${ }^{13}$ Details of the study design and a previous analysis using the same dataset have been published. ${ }^{14}$ Data were collected through an extensive postal questionnaire on smoking, occupational exposure (including fibres, fumes, gas, mineral dust, organic dust and vapours) and diagnosis of PF. The questionnaire has been described in detail elsewhere. ${ }^{15}$ Smoking data included the year of starting smoking, date of stopping smoking and the mean number of smoked cigarettes per day during each 10 -year period between ages 15 and 65 years, and the mean exposure after age $65 .{ }^{15}$ Occupational data included the presence, start year, stop year and intensity (h/week) for any occupational exposure and exposure to birds (at work and at home), metal dust and wood dust. Occupational exposure was defined as any exposure 10 or more years before the date of the PF diagnosis, allowing for a 10-year time lag between exposures and developing the disease.

After exclusion of patients who were incorrectly registered $(\mathrm{n}=12 ; 5 \%)$ or did not respond to the questionnaire $(\mathrm{n}=58 ; 24 \%), 171 \mathrm{PF}$ cases were included in the analysis.

Patients with probable IPF (IPF cases, $n=137$ ) were identified through a review of each patient's medical record performed independently by specialists in respiratory medicine (KN, TG) ${ }^{14}$ High-resolution CT was performed in $41 \%$ of patients with PF, CT in an additional $10 \%$ and transbronchial and open lung biopsy was performed in $6 \%$ of the patients. The IPF cohort excluded patients with an identifiable or probable cause of PF: rheumatic or systemic inflammatory diseases (20\% of PF cases), pneumoconiosis $(6 \%)$, and medications or irradiation $(2 \%) .{ }^{14}$

Control participants were selected as a random sample $(n=1000)$ from the general population of the same age range as the patients with $\mathrm{PF}$. Of the control participants, $719(72 \%)$ returned complete exposure data and were included in the analysis. All participants gave their informed consent to participate.

\section{Statistical analysis}

Cases and control participants were categorised according to year of birth (1906-1923, 1924-1936 or 1937-
1969) and cases according to the year they received their PF diagnosis (1968-1986, 1987-1993 or 19941999). Control participants were assigned a time point corresponding to the year the patients received their PF diagnosis, using a method described previously. ${ }^{14}{ }^{15}$ First, control participants within each birth year group were assigned a random diagnosis year group, weighted by the number of cases in each diagnosis year group. Then, the year of PF diagnosis of each control participant was set to the mid-year of the corresponding year of the patient group. Characteristics at baseline (the date the questionnaire was filled in) were presented using frequencies and percentages for categorical variables. Continuous data were presented using mean with $\mathrm{SD}$ and median with range or IQR for variables with normal and skewed distribution, respectively.

Smoking status and cumulative smoking exposure, calculated as pack-years ((mean number of cigarettes per day) $/ 20 \times($ years of exposure $)$, were recorded up to 10 years prior to the date of the PF diagnosis (a 10-year time lag), allowing for time between smoke exposure and the development of oxygen-dependent PF.

Associations between smoking and the risk of developing oxygen-dependent PF and IPF, and the interactions between smoking, occupational exposure and gender, were estimated and reported using Mantel-Haenszel analysis, controlling for year of birth, year of diagnosis and gender, as applicable. Interactions were also analysed using conditional logistic regression, stratified for year of birth, year of diagnosis, gender, with adjustment for age and pack-years of smoking. All estimates were consistent between Mantel-Haenszel analysis and the conditional logistic models.

Associations were expressed as ORs with 95\% CIs. Statistical significance was defined as a double-sided $\mathrm{p}<0.05$. Statistical analyses were performed using Stata V.11.1 (StataCorp LP; College Station, Texas, USA) and SAS V.9.2 (SAS Institute, Inc, Cary, North Carolina, USA).

\section{RESULTS}

We included 171 patients with PF, of whom 137 were classified as having IPF, and 719 control participants. Baseline characteristics are shown in table 1. Among PF cases, the rate of any occupational exposure was higher in men than in women ( $80 \%$ vs $52 \%$; $<<0.001)$. Men also had higher smoking exposure. Ten years before the PF diagnosis, $90(84 \%)$ men were ever-smokers with a median of 10 (IQR, 3-23) pack-years, compared with 29 (45\%) women with a median of 8 (IQR, 3-15) packyears. A similar difference was seen in IPF cases.

\section{Interactions}

There was a significant interaction between smoking, occupational exposure and gender and the risk of developing oxygen-dependent PF (test of homogeneity, $\mathrm{p}=0.028$ ). The interaction was similar in the IPF cohort 
Table 1 Patient characteristics

\begin{tabular}{lccc}
\hline Characteristics & PF cases $(\mathbf{n = 1 7 1 )}$ & IPF cases $(\mathbf{n = 1 3 7 )}$ & Controls $(\mathbf{n = 7 1 9 )}$ \\
\hline Age & $73.7 \pm 9.5$ & $74.2 \pm 9.8$ & $64.3 \pm 13.7$ \\
Males, $n(\%)$ & $107(63)$ & $86(63)$ & $337(47)$ \\
Never-smokers, $n(\%)$ & $52(30)$ & $44(32)$ & $344(48)$ \\
Ex-smokers, $n(\%)$ & $114(67)$ & $89(65)$ & $251(35)$ \\
Current smokers, $n(\%)$ & $5(3)$ & $4(3)$ & $124(17)$ \\
Smoking exposure, $n(\%)^{*}$ & $119(70)$ & $93(68)$ & $375(52)$ \\
$1-9$ pack-years & $29(17)$ & $22(16)$ & $176(24)$ \\
$10-19$ pack-years & $34(20)$ & $27(20)$ & $91(13)$ \\
$\geq 20$ pack-years & $36(21)$ & $27(20)$ & $62(9)$ \\
Occupational exposure, $n(\%)^{*}$ & $119(70)$ & $93(68)$ & $397(55)$ \\
Birds & $16(9)$ & $11(8)$ & $33(5)$ \\
Inorganic dust & $55(32)$ & $40(29)$ & $164(23)$ \\
Metal dust & $35(20)$ & $27(19)$ & $119(17)$ \\
Organic dust & $67(39)$ & $52(38)$ & $182(25)$ \\
Wood dust & $32(18)$ & $25(18)$ & $57(8)$ \\
\hline Data presented as mean $\pm S D$ unless otherwise specified. & & \\
*Exposure earlier than 10 years before PF diagnosis (10-year time lag). & & \\
IPF, idiopathic pulmonary fibrosis; PF, pulmonary fibrosis. & &
\end{tabular}

(table 2). Men with current or past smoking and occupational exposure had markedly increased risk of PF, OR 4.6 (2.1 to 10.3), and IPF, OR 3.0 (1.3 to 6.5), compared with non-exposed women (table 2). Adjustment for differences in pack-years between men and women, in addition to the other covariates, did not affect the estimates.

\section{Dose-response effect}

There was a linear association between higher cumulative smoking exposure (up to 10 years before diagnosis) and increased risk of PF and IPF, OR 1.03 (95\% CI 1.01 to 1.04) per pack-year and OR 1.02 (95\% CI 1.01 to 1.04) per pack-year, respectively. Compared with lower levels of smoking (1-9 pack-years), heavy smoking ( $\geq 20$ pack-years) was associated with an increased risk of PF, OR 2.6 (95\% CI 1.4 to 4.9) and IPF, OR 2.5 (95\% CI 1.3 to 5.0), as shown in table 3. Using a 5-year time lag for smoking exposure instead of 10 years resulted in similar estimates.

\section{Subtypes of occupational exposure}

The effect of occupational exposure seemed to be mediated partly through exposure to birds and wood dust. The risk of PF was increased by exposure to birds (OR 1.9; 95\% CI 1.0 to 3.7) and wood dust (OR 1.7;
95\% CI 1.0 to 3.0 ), controlling for age, gender, year of diagnosis and smoking. There were no evidence of effects of inorganic dust (OR 1.3; 95\% CI 0.8 to 2.0) or metal dust (OR 1.1; 95\% CI 0.6 to 1.8 ). There were signs of interactions with smoking and gender for exposure to birds $(p=0.021)$ and wood dust $(p=0.023)$, respectively. Estimates were similar for the IPF cohort, except for a lower effect of bird exposure (OR 1.3; $95 \%$ CI 0.6 to 2.8 ).

\section{DISCUSSION}

The main findings are that (1) smoking was a risk determinant in the development of oxygen-dependent PF and that this risk was amplified by male gender and occupational exposure; (2) the association with smoking was dose-dependent, which may support the theory of the causative role of smoking in the pathogenesis of severe PF.

Our findings are consistent with reports of increased risk of IPF associated with smoking ${ }^{4-6} 101216$ and occupational exposures. $^{4-7} 9121416$ A previous analysis using the present dataset showed that specific occupational factors associated with an increased risk of PF included exposure to birds and wood dust. ${ }^{14}$ Studies of a possible

Table 2 Effect of smoking on the adjusted risk of pulmonary fibrosis, according to gender and occupational exposure

\begin{tabular}{|c|c|c|c|c|}
\hline & \multicolumn{2}{|l|}{ PF OR (95\% Cl) } & \multicolumn{2}{|l|}{ IPF OR (95\% CI) } \\
\hline & Women & Men & Women & Men \\
\hline No occupational exposure & 1.10 (0.50 to 2.42$)$ & 1.97 (0.64 to 6.13$)$ & $1.12(0.49$ to 2.59$)$ & $1.44(0.43$ to 4.83$)$ \\
\hline Occupational exposure & $1.10(0.52$ to 2.34$)$ & 4.63 (2.08 to 10.33$)$ & $1.32(0.58$ to 3.03$)$ & 2.96 (1.34 to 6.52$)$ \\
\hline
\end{tabular}


Table 3 Dose-response effect of smoking on the risk of severe pulmonary fibrosis

\begin{tabular}{lll}
\hline Smoking, pack-years ${ }^{*}$ & PF OR (95\% CI) & IPF OR (95\% CI) \\
\hline 0 & 1 & 1 \\
$1-9$ & $1.03(0.62$ to 1.70$)$ & $0.90(0.52$ to 1.57$)$ \\
$10-19$ & $2.26(1.35$ to 3.80$)$ & $2.10(1.20$ to 3.68$)$ \\
$\geq 20$ & $2.66(1.56$ to 4.55$)$ & $2.25(1.26$ to 4.02$)$ \\
\hline ORs for levels of smoking estimated using conditional logistic regression adjusted for age and stratified for year of birth, year of diagnosis, \\
gender and occupational exposure. \\
"Pack-years of smoking up to 10 years before the year of PF diagnosis. \\
IPF, idiopathic pulmonary fibrosis; PF, pulmonary fibrosis.
\end{tabular}

dose-response correlation between smoking and IPF have shown conflicting results, with two studies indicating a dose-dependent effect ${ }^{5} 6$ and one study showing no dose correlation. ${ }^{17}$ The latter study, however, analysed only smoking status and the current smoking dose (cigarettes per day) and not cumulative smoking exposure such as pack-years. ${ }^{17}$ The present study extends the previous observations by demonstrating that the association between smoking and severe PF is dose-dependent and is modified by gender and occupational exposure.

The strength of the present study is that it included cases from a population-based prospective register of patients starting LTOT in Sweden. Control participants were randomly selected from the general population. Previous studies using control participants in hospitals may have yielded biased estimates, as the risk of hospitalisation is likely to be related to occupational factors and smoking. ${ }^{7}$ 9-11 We had detailed data on the temporality, dose and duration of smoking. In contrast with previous studies, only exposure data up to 10 years before the year of the PF diagnosis was included in the analysis to avoid reverse causation and to allow for the time lag between exposure to risk factors and the manifestation of clinical disease.

A possible limitation is that the self-reported exposure data could be influenced by recall bias. The validity of the exposure classification was, however, supported by a high degree of consistency between reported employment histories and occupational exposure to specific agents. ${ }^{14}$ Second, the association between smoking and starting LTOT could be affected by survivor bias, as smokers are likely to be at high risk of dying of other smoke-related disease, such as cancer and cardiovascular disease, before they can develop severe IPF. Also, stopping smoking is a mandatory criterion for starting LTOT. Both these potential biases would tend to lower the number of smokers starting LTOT and to underestimate the association between smoking and oxygen-dependent PF. Third, the IPF diagnosis could be misclassified in some patients, especially as the cohort was collected prior to the publication of main consensus definition of IPF. ${ }^{1}$ The validity of the PF and IPF diagnoses was checked by respiratory physicians using medical records, including available radiographic and histological data. ${ }^{14}$ Among idiopathic interstitial pneumonias, IPF is the most common condition and it is associated with a high risk of progression to hypoxemic respiratory failure and death. $^{1} 18$ Thus, the prevalence of IPF is most likely to be high in oxygen-dependent PF. It is possible that we included patients with combined PF and emphysema, which may be present in up to one-third of patients with IPF. $^{19}$ Concurrent emphysema may constitute a smoking-related comorbidity or a distinct IPF phenotype, ${ }^{19}$ and could explain, at least partly, the association between smoking, male gender and the development of severe IPF in the present study. We included the PF cohort in the analysis, as it may be difficult to obtain a specific diagnosis in patients with advanced PF in the clinic. Findings were similar in the PF and IPF cohorts, which supports the validity of the analysis. Using national population-based cases and controls, the present findings most likely have high applicability to severe PF in Swedish clinical practice. The validity to other settings may be lower owing to differences in sociodemographic factors, healthcare organisation and pattern of exposure.

Mechanisms governing the relationship between smoking, gender, occupational exposure and the development of severe PF are unknown but likely involve complex interactions between different environmental factors in genetically predisposed individuals. ${ }^{20}$ The adverse effect of smoking could in part be attributable to the development of concurrent emphysema, which has been associated with hypoxaemia and earlier death in IPF. $^{21}$

For the clinicians, this study identifies a group of male, heavy smokers with occupational exposure to harmful substances, who have a greatly increased risk of developing severe PF. In this group, interventions to help people reduce or stop smoking are a top priority.

In conclusion, smoking is associated with a dosedependent increase in oxygen-dependent PF. The adverse effects of smoking are stronger in men and in people with occupational exposure.

\section{Author affiliations}

${ }^{1}$ Department of Clinical Sciences, Division of Respiratory Medicine \& Allergology, Lund University, Lund, Sweden

${ }^{2}$ Department of Medicine, Blekinge Hospital, Karlskrona, Sweden

${ }^{3}$ Institution of Public Health and Clinical Medicine, Umeå University, Umeå, Sweden 
${ }^{4}$ Respiratory Medicine Unit, Department of Medicine Solna, The Karolinska Institute and Karolinska University Hospital, Stockholm, Sweden

${ }^{5}$ Section for Occupational Medicine, Respiratory Diseases and Toxicology, University of Perugia, Perugia, Italy

${ }^{6}$ Section for Occupational and Environmental Medicine, Institute of Medicine, Sahlgrenska Academy at the University of Gothenburg, Gothenburg, Sweden

Acknowledgements All the authors would like to thank Kerstin Ström for her invaluable help and inspiration throughout the study process, and the doctors and nurses who cared for the patients.

Contributors ME had full access to all the data in the study and took full responsibility for the integrity of the data and the accuracy of the data analysis. KB, KN, GT and KT were involved in conception and design. TG, KB $\mathrm{KN}$, GT and KT were involved in the acquisition of the data. ME, TG, NM and $\mathrm{KT}$ were involved in analysis and interpretation of data. ME, TG and KT were involved in drafting the article. ME, TG, KB, KN, GT, NM and KT were responsible for revising the article for important intellectual content and approval of the version to be published.

Funding This study was funded by the Research Council of Blekinge, the Swedish National Board of Health and Welfare and the Swedish Heart-Lung Foundation.

Competing interests None.

Ethics approval The study was approved by all the relevant ethics committees in Sweden, the Swedish National Board of Health and Welfare, and the Data Inspection Board. Ethics committée of Lund University, Sweden.

Provenance and peer review Not commissioned; externally peer reviewed.

Data sharing statement Analysis code is available from the corresponding author.

Open Access This is an Open Access article distributed in accordance with the Creative Commons Attribution Non Commercial (CC BY-NC 3.0) license, which permits others to distribute, remix, adapt, build upon this work noncommercially, and license their derivative works on different terms, provided the original work is properly cited and the use is non-commercial. See: http:// creativecommons.org/licenses/by-nc/3.0/

\section{REFERENCES}

1. American Thoracic Society/European Respiratory Society International Multidisciplinary Consensus Classification of the Idiopathic Interstitial Pneumonias. This joint statement of the American Thoracic Society (ATS), and the European Respiratory Society (ERS) was adopted by the ATS board of directors, June 2001 and by the ERS Executive Committee, June 2001. Am J Respir Crit Care Med 2002;165:277-304.

2. Raghu G, Collard HR, Egan JJ, et al. An official ATS/ERS/JRS/ ALAT statement: idiopathic pulmonary fibrosis: evidence-based guidelines for diagnosis and management. Am J Respir Crit Care Med 2011;183:788-824.

3. Navaratnam V, Fleming KM, West $\mathrm{J}$, et al. The rising incidence of idiopathic pulmonary fibrosis in the U.K. Thorax 2011;66:462-7.

4. Iwai K, Mori T, Yamadaa N, et al. Idiopathic pulmonary fibrosis. Epidemiologic approaches to occupational exposure. Am J Respir Crit Care Med 1994;150:670-5.

5. Hubbard R, Lewis S, Richards K, et al. Occupational exposure to metal or wood dust and aetiology of cryptogenic fibrosing alveolitis. Lancet 1996;347:284-9.

6. Baumgartner KB, Samet JM, Stidley CA, et al. Cigarette smoking: a risk factor for idiopathic pulmonary fibrosis. Am J Respir Crit Care Med 1997; 155:242-8.

7. Miyake Y, Sasaki S, Yokoyama T, et al. Occupational and environmental factors and idiopathic pulmonary fibrosis in Japan. Ann Occup Hyg 2005;49:259-65.

8. Scott J, Johnston I, Britton J. What causes cryptogenic fibrosing alveolitis? A case-control study of environmental exposure to dust. BMJ 1990;301:1015-17.

9. Awadalla NJ, Hegazy A, Elmetwally RA, et al. Occupational and environmental risk factors for idiopathic pulmonary fibrosis in Egypt: a multicenter case-control study. Int J Occup Environ Med 2012;3:107-16.

10. Garcia-Sancho Figueroa MC, Carrillo G, Perez-Padilla R, et al. Risk factors for idiopathic pulmonary fibrosis in a Mexican population. A case-control study. Respir Med 2010;104:305-9.

11. Miyake $\mathrm{Y}$, Sasaki S, Yokoyama T, et al. Case-control study of medical history and idiopathic pulmonary fibrosis in Japan. Respirology 2005;10:504-9.

12. Taskar VS, Coultas DB. Is idiopathic pulmonary fibrosis an environmental disease? Proc Am Thorac Soc 2006:3.293-8.

13. Strom K, Boe J. A national register for long-term oxygen therapy in chronic hypoxia: preliminary results. Eur Respir J 1988;1: 952-8.

14. Gustafson T, Dahlman-Hoglund A, Nilsson $\mathrm{K}$, et al. Occupational exposure and severe pulmonary fibrosis. Respir Med 2007;101:2207-12.

15. Toren K, Jarvholm B, Brisman J, et al. Adult-onset asthma and occupational exposures. Scand J Work Environ Health 1999;25:430-5.

16. Baumgartner KB, Samet JM, Coultas DB, et al. Occupational and environmental risk factors for idiopathic pulmonary fibrosis: a multicenter case-control study. Collaborating Centers. $\mathrm{Am} \mathrm{J}$ Epidemiol 2000;152:307-15.

17. Gribbin J, Hubbard R, Smith C. Role of diabetes mellitus and gastro-oesophageal reflux in the aetiology of idiopathic pulmonary fibrosis. Respir Med 2009;103:927-31.

18. Bjoraker JA, Ryu JH, Edwin MK, et al. Prognostic significance of histopathologic subsets in idiopathic pulmonary fibrosis. Am J Respir Crit Care Med 1998;157:199-203.

19. Cottin V. The impact of emphysema in pulmonary fibrosis. Eur Respir Rev 2013;22:153-7.

20. Macneal K, Schwartz DA. The genetic and environmental causes of pulmonary fibrosis. Proc Am Thorac Soc 2012;9:120-5.

21. Mejia M, Carrillo G, Rojas-Serrano J, et al. Idiopathic pulmonary fibrosis and emphysema: decreased survival associated with severe pulmonary arterial hypertension. Chest 2009;136:10-15. 